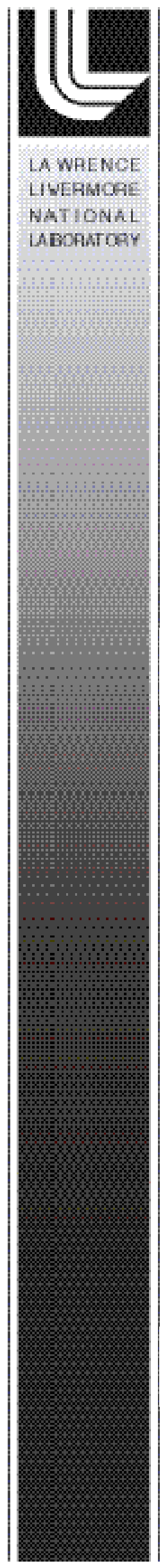

UCRL-TR-205798

FLASH X-RAY (FXR) ACCELERATOR OPTIMIZATION Beam-induced Voltage Simulation and TDR Measurements

Mike M. Ong and George E. Vogtlin

April 5, 2004 


\section{Disclaimer}

This document was prepared as an account of work sponsored by an agency of the United States Government. Neither the United States Government nor the University of California nor any of their employees, makes any warranty, express or implied, or assumes any legal liability or responsibility for the accuracy, completeness, or usefulness of any information, apparatus, product, or process disclosed, or represents that its use would not infringe privately owned rights. Reference herein to any specific commercial product, process, or service by trade name, trademark, manufacturer, or otherwise, does not necessarily constitute or imply its endorsement, recommendation, or favoring by the United States Government or the University of California. The views and opinions of authors expressed herein do not necessarily state or reflect those of the United States Government or the University of California, and shall not be used for advertising or product endorsement purposes.

This work was performed under the auspices of the U.S. Department of Energy by University of California, Lawrence Livermore National Laboratory under Contract W-7405-Eng-48. 


\title{
FLASH X-RAY (FXR) ACCELERATOR OPTIMIZATION Beam-induced Voltage Simulation and TDR Measurements
}

\author{
Mike M. Ong and George E. Vogtlin \\ Lawrence Livermore National Laboratory, PO Box 808, L-153 \\ Livermore, CA, 94550
}

\section{Summary}

Lawrence Livermore National Laboratory (LLNL) is evaluating design alternatives to improve the voltage regulation in our Flash X-Ray (FXR) accelerator cell and pulse-power system. The goal is to create a more mono-energetic electron beam that will create an x-ray source with a smaller spot-size. Studying the interaction of the beam and accelerator cell will generate improved designs for high-current accelerators at Livermore and elsewhere.

When an electron beam crosses the energized gap of an accelerator cell, the electron energy is increased. However, the beam with the associated electromagnetic wave also looses a small amount of energy because of the increased impedance seen across the gap. The phenomenon is sometimes called beam loading. It can also be described as a beam-induced voltage at the gap which is time varying. This creates beam energy variations that we need to understand and control.

A high-fidelity computer simulation of the beam and cell interaction has been completed to quantify the time varying induced voltage at the gap. The cell and pulse-power system was characterized using a Time-domain Reflectometry (TDR) measurement technique with a coaxial air-line to drive the cell gap. The beam-induced cell voltage is computed by convoluting the cell impedance with measured beam current. The voltage was checked against other measurements to validate the accuracy.

The simulation results predicted that there are significant beam-induced gap voltage variations. Beam-induced voltages from different current profiles and cell impedances were simulated and compared. This allows us to predict the effect on voltage regulation for different design alternatives before making hardware changes and high-voltage testing.

The beam-induced voltages are incorporated into a larger accelerator system-model to quantify their effect on total beam energy variations.

\section{FXR Energy Regulation and Test Stand}

The LLNL FXR is an induction linear accelerator that produces pulsed x-rays and is used regularly and reliably on explosive experiments since its completion in 1982. In recent years FXR has been incrementally improved, adding double-pulse capability, increasing dose, and reducing x-ray spot-size [1].

The accelerator generates a $3 \mathrm{kA}$ electron beam with $17 \mathrm{MeV}$ of energy. Our present pulse length is about $70 \mathrm{~ns}$. The x-ray dose at $1 \mathrm{~m}$ is over $400 \mathrm{Rad}$, and the current spot-size is $2 \mathrm{~mm}$ (full-width half-maximum).

Based on comparison of dose and x-ray spot-size from the Los Alamos National Laboratory (LANL) DARHT and other accelerators, we believe that we could further improve the performance of FXR. In 2001, during the construction of a very large explosive containment facility, the accelerator was not needed for hydrodynamic experiments. We had the opportunity to study the limitations of FXR along with colleagues from the LLNL Beam Research Program.

Results from a measurement campaign and computer modeling of the electron beam at the final focus magnet identified two areas of improvement that might significantly reduce the $\mathrm{x}$-ray spot-size: beam energy regulation and beam emittance.

There are two sources of beam energy variations: the pulse-power system and the beam interaction with the cell and pulse-power system. The first two terms of the target energy equation (1) includes the voltage that is generated by the Marx and Blumlein, along with their interactions with the time-isolation and power feed coaxial lines and cell features. The injector voltage has added complexity because of the reflections in the cathode and anode stalks. The third term is defined as the beam-induced gap voltage that launches an electromagnetic (EM) wave into the cell and pulse-power system. A portion is reflected back from the different cell components and appears in the gap again. This is related to beam loading, but the impedance mismatches in the cell and pulse-power system creates a much more dynamic process than the name "loading" implies. This report focuses on the beam-induced energy variation.

$$
E_{V \text { injector }}+E_{V \text { accelerator }}-E_{V \text { beam-induced }}=E_{\text {target }}
$$


Because of the limitation of the computers and codes in the 1980's, the voltage variation problems were not fully modeled when the accelerator was designed more than 20 years ago. Now, we have better modeling capabilities, diagnostic equipment, and design experience gained from the development of other accelerators. We know that both the pulse-power system and accelerator cell can be improved. The goal for the optimization project is to generate a wellregulated voltage pulse of sufficient length and transport it to the cell while minimizing early reflections. This should also diminish reflections associated with the beam-induced pulse.

Experimenting with an expensive and important production machine like FXR by changing components is not acceptable. It is even difficult to diagnose the FXR accelerator because of the busy experimental shot schedule. Nonetheless, a limited number of measurements have been completed to characterize the performance of the FXR system.

Ray Scarpetti decided that alternative designs for improving voltage regulation could not be evaluated on FXR. Instead, he directed the construction a Single-cell Test Stand that would allow new designs to be studied without interfering with the shot schedule or jeopardizing FXR reliability [2].

The goal of the Test Stand studies is to generate a voltage pulse at the acceleration gap that varies by considerably less than $1 \%$-rms for a 70 ns duration that comfortably encompasses most $(60 \mathrm{~ns})$ of the beam. This must include the contribution from the beam-induced voltage.

Wherever possible, spare FXR parts were used in the construction of the Test Stand. The cell is an unused unit. The Marx generator normally feeds four Blumleins/cells, but the one for the Test Stand was modified to fire into only one cell. The Stand was constructed in the high-bay of the FXR accelerator so that the auxiliary systems (such air conditioning, water cooling, high dielectric-strength oil, deionized water, electrical power, and vacuum) could be provided by existing FXR equipment.

Two approaches were proposed to quantify the beaminduced voltages in the cell: (1) develop a full-current dynamic beam-load simulator and (2) make a low-voltage TDR measurement. TDR results would also be used in computer simulations to quickly evaluate different design options.

The concept for the dynamic beam-load simulator is shown in Figure 1. A metal rod runs through the cell in place of the beam. One end of the rod is grounded. The other is attached to one side of a high-voltage liquid load resistor. The other end of the resistor is connected to ground through a triggered switch gap. This would allow the simulated "beam" to be delayed. The current-induced voltage can be measured at the load resistor.

Low-voltage TDR measurements provide higher fidelity data. Identification of cell components that generate the time varying induced gap voltages is possible. By opening ports we can safely and easily insert shorting bars to associate features in the TDR voltage waveform with cell locations.

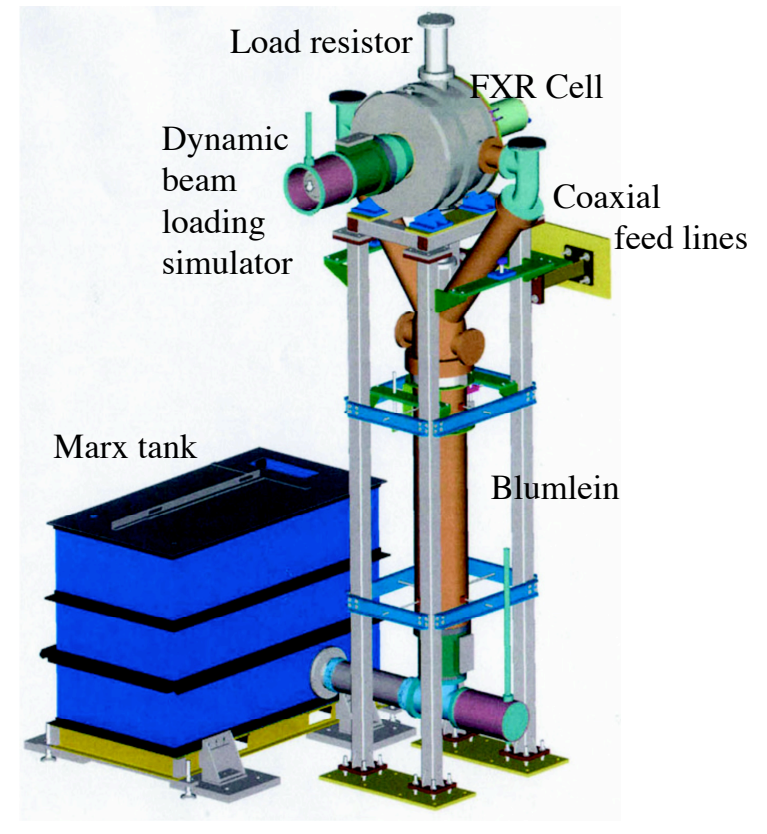

Figure 1. A drawing of the FXR Single-cell Test Stand shows the pulse-power system, cell, and proposed beam load simulator to evaluate new designs with improved voltage regulation.

\section{TDR Measurements}

The low-voltage approach for studying beam-induced potentials is shown in Figure 2. A $50 \Omega$ air-line is attached to the cell. The air-line is driven from the right with the pulser of a Time-domain Reflectometry system. This emulates effect of the electron beam passing through the cell. The other end of the air-line is terminated with a $50 \Omega$ load. While the impedance between the beam and beam-pipe varies, the $50 \Omega$ test components were chosen because $50 \Omega$ pulsers, cables, and terminations are readily available. The effect of the $50 \Omega$ impedance of the airline will be removed from the measurements to obtain the cell impedance. 


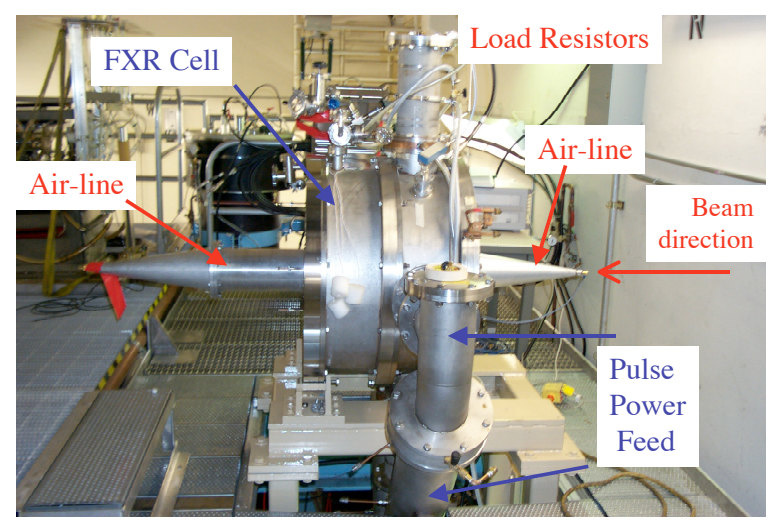

Figure 2. A $50 \Omega$ air-line excites the cell in the longitudinal direction to measure the cell gap impedance.

The radius of the outer conductor of the air-line has the same radius as our beam pipe-in the cell. (See Figure 3.) The air-line design is given by the following formula:

$$
\begin{aligned}
& Z=60 \ln \underset{\text { radius }_{\text {outer }}}{\text { radius }_{\text {inner }}} \\
& Z=60 \ln \stackrel{\square 2.85^{\prime \prime}}{\square} \stackrel{\square}{\square}=50 \square
\end{aligned}
$$

The ends of the air-line are tapered to accept an "N"-type connector.

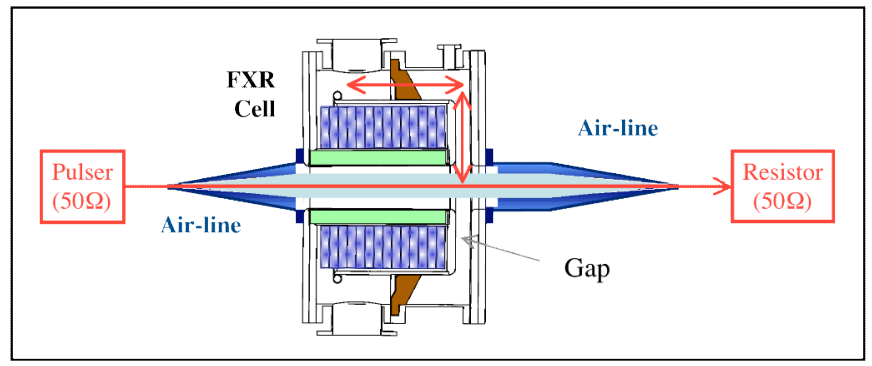

Figure 3. A cross section of the cell interior and air-line shows the power flow from the pulser and reflections.

When TDR measurements are taken, the cell is connected to the pulse power system and filled with the normal fluids, water and oil. For convenience, the cell is not evacuated because the dielectric constant of air and vacuum is the same, essentially one.

The TDR instrument is shown in Figure 4. The rise- time of the pulse is about 50 ps. This is more than adequate because the FXR beam rise-time is much slower, about 10 ns.

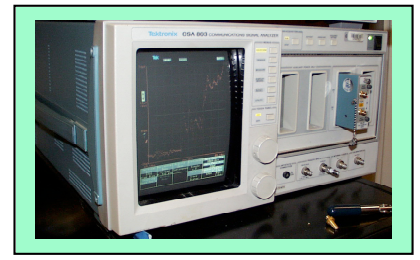

Figure 4. The Tektronix CSA803 TDR has a $0.5 \mathrm{~V}$ pulser with a fast rise-time of $50 \mathrm{ps}$.

The measurement process begins with the TDR pulser sending an incident step wave into the cable that is connected to the air-line. (See Figure 5.) When the edge of the step passes the cell gap, a portion of the voltage is reflected back towards the pulser because of the impedance change. The reflected wave is recorded on a sampling oscilloscope with high-input impedance. The portion of the step that is not reflected is absorbed by the $50 \Omega$ load at the right.

The impedance at the cell is determined by the following formula:

$$
\frac{V_{\text {reflected }}}{V_{\text {incident }}}=\frac{Z_{\text {cell }+ \text { airline }}-Z_{50 \square}}{Z_{\text {cell }+ \text { airline }}+Z_{50 \square}}
$$

where the air-line impedance is $50 \Omega$. The distance to the cell component that caused the impedance change can be determined from the timing of the reflected pulse.

The measured cell impedance is shown at the bottom of Figure 6. The first two peaks are created in the acceleration gap (designated by green arrow) and corner (blue arrow). The back wall impedance is located between $5 \mathrm{~ns}$ and $6 \mathrm{~ns}$ and appears to be a short.

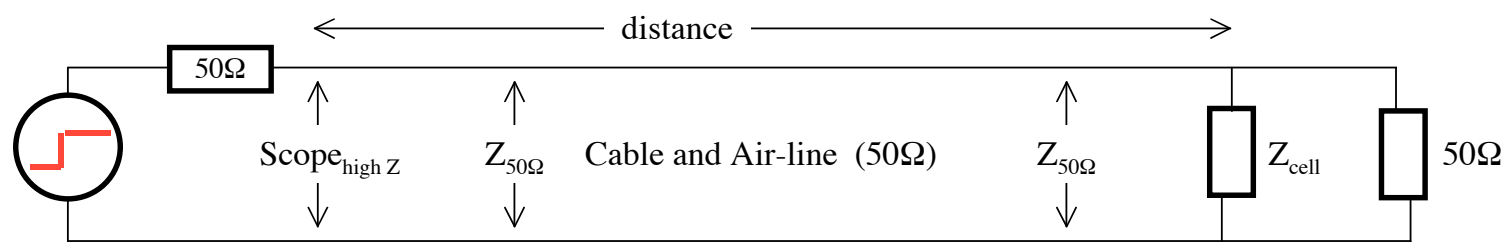

Figure 5. Time-domain Reflectometry can be used to determine the impedance of the cell. 


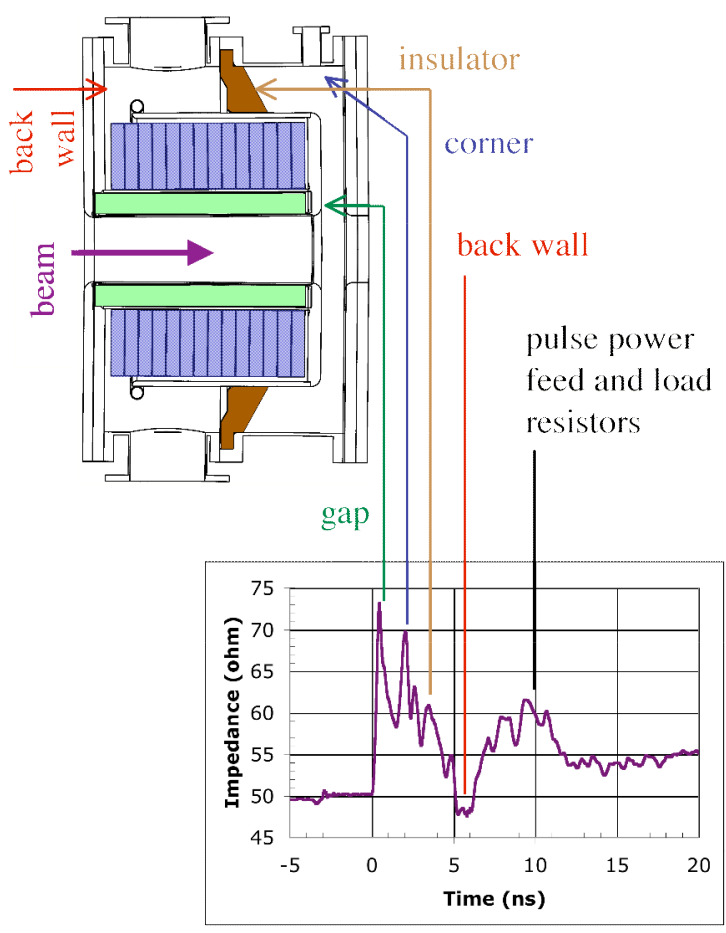

Figure 6. Cell impedance as a function of time starting at the gap.

The measured impedances agree reasonably well with levels computed analytically. The area between the gap and corner is normally under vacuum, and the impedance can be estimated from the formula for parallel plates transmission lines. (See Figure 7.) The two computed impedances must be added to the $50 \Omega$ of the air-line to equal the TDR results.

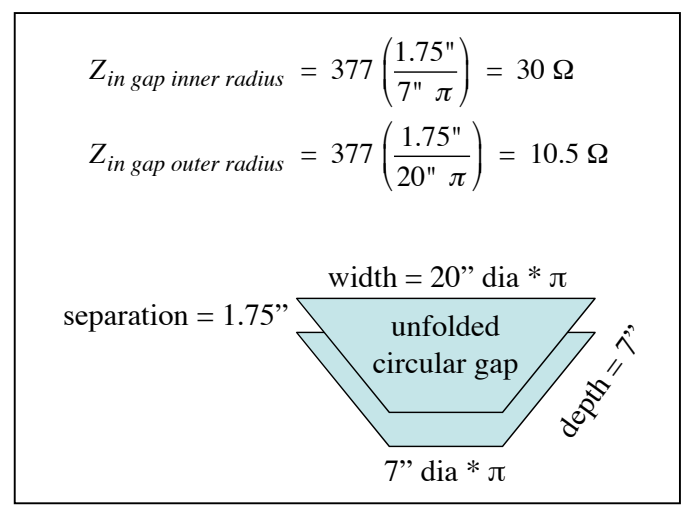

Figure 7. The impedance in the cell gap area can be estimated from parallel plate transmission theory.

The cylindrical space between the corner and back wall has a coaxial geometry. The following formula can be used to estimate the impedance.

$$
Z=\frac{1}{2 \square} \sqrt[\square]{\square} \ln {\sqrt{r_{\text {inner }}}}_{=}^{=}
$$

The impedance in the insulator and the space between the insulator and back wall filled with oil is lower because of the higher dielectric constant.
A longer time record of the cell impedance is shown in Figure 8. The flat portion from about $25 \mathrm{~ns}$ to $95 \mathrm{~ns}$ is generated by the water-lines and load resistors. The water-line provides time isolation between the cell and the high-voltage Blumlein pulse generator. The measured impedance in the flat region is $56 \Omega$. The two water-lines in parallel have $10.7 \Omega$ impedance. The two load resistors located in the cylinders above and below the cell are $44 \Omega$ each. The combined impedance in parallel is $7.2 \Omega$. This is reasonably close to the $6 \Omega(56 \Omega-50 \Omega)$ from the TRD measurements. The bump at the end between $95 \mathrm{~ns}$ and 100 $\mathrm{ns}$ is near the top of the Blumlein.

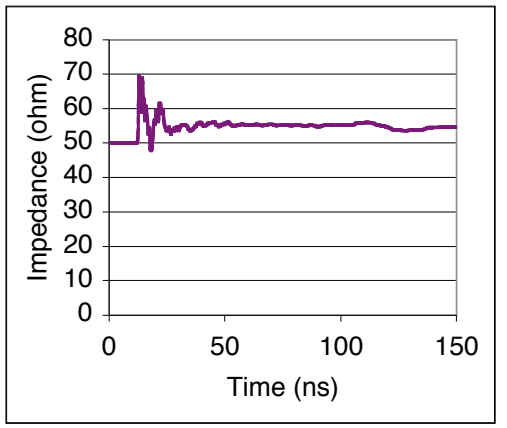

Figure 8. The impedance of the water-line for time isolation is shown in the middle of the plot.

The step waveform from the TDR pulser has significant droop for $150 \mathrm{~ns}$. The impedance in Figure 8 has been droop corrected. The droop was measured and a correction was applied to the raw voltage measurements. Corrected cell impedances are used to compute the gap voltage when excited by the beam.

\section{Computer Simulation of Beam-induced Cell Voltage}

The gap voltage is computed by convolving the impulse cell response (see right plot of Figure 9) with the beam current (see left plot).

$\mathrm{V}_{\text {gap-induced }}(\mathrm{t})=\left[\mathrm{I}_{\text {beam }} * \mathrm{Z}_{\text {impulse-gap }}\right](\mathrm{t})=\int I(\square \mathrm{Z}(t-\square d \square$

The TDR instrument excites the cell with a step function. To obtain the impulse response of the cell, the step response is differentiated. The result is shown in the right plot of Figure 9.
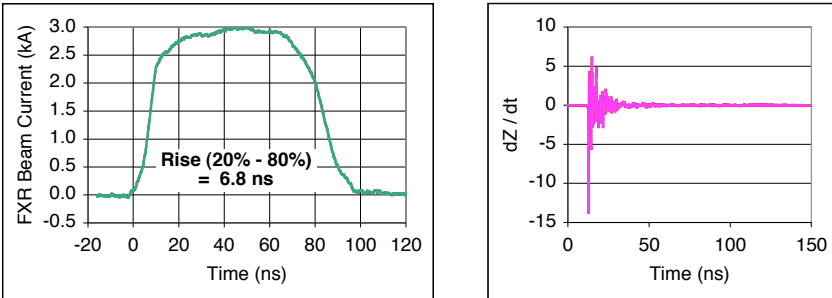

Figure 9. Beam current and impulse response of FXR cell derived from TDR measurements. 
The current was measured with resistive-wall beam sensors, also known as beam-bugs. The rise-time of the e-beam is $6.8 \mathrm{~ns}$ from $20 \%$ to $80 \%$. These current levels were chosen because the traditional $10 \%$ to $90 \%$ points show an unrealistic slow rise-time. The equivalent rise-rate is $9 \mathrm{~ns}$ if we extend the same slope to the $10 \%$ and $90 \%$ levels.

The convolution was performed with MATLAB. The sample interval for the beam current was $0.2 \mathrm{~ns}$, which very accurately represents the rise-time. The TDR data was taken at $40 \mathrm{ps}$ intervals, and de-sampled to $0.2 \mathrm{~ns}$. Low-pass filtering greatly reduced the random noise in the measured reflected voltage and allowed a more accurate differentiation.

The simulated gap voltage is shown in Figure 10 and is overlaid with the current. The voltage is really negative and has the opposite polarity of the accelerating voltage.

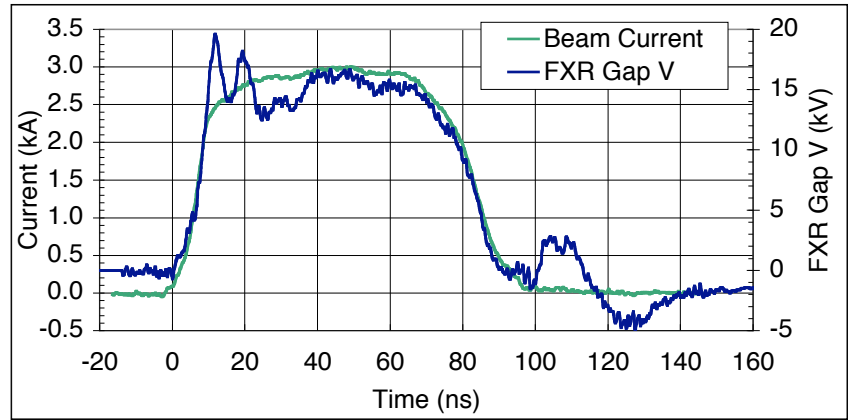

Figure 10. Beam current and computed beam-induced gap voltage have the same duration.

There are some fast and slow oscillations occurring during the first third of the beam. This variation is a major contributor to beam energy variation that increases $\mathrm{x}$-ray spot-size. These oscillations are caused by impedance changes identified in the previous section. The impedance mismatches in the cell and pulse power system creates voltage reflections that propagate back to the acceleration gap. Hence, they cause the beam energy to change. This effect occurs at every cell. So, the beam energy should vary like the induced-voltage, and this will be demonstrated in the validation sub-section.

Ideally, the voltage profile would be the same as the current profile if the impedance was a constant 7.2 $\Omega$. Based on theory, the induced voltage at the middle of the beam should be $22 \mathrm{kV}$ ( $3 \mathrm{kA} \times 7.2 \Omega$ ). The simulation predicted $17 \mathrm{kV}$ because the measured "steady-state" impedance was about $6 \Omega$. This beam loading causes the acceleration voltage to drop about $5 \%$.

\section{Validation}

The validity of the simulations was checked against three types of measurements: (1) energy analyzer at the end of the accelerator, (2) cell voltage from pulse-power system with and without beam, and (3) beam-induced voltage in the cell. They all support the simulation results.
(1) In the spring of 2000, LANL loaned FXR their energy analyzer [3]. The analyzer was installed in the drift section after the accelerator. A carbon collimator blocked most of the FXR beam, and a precision magnet bent the beamlet that was allowed to pass. The electrons were converted to light photons with a fast scintillator. The vertical position of the spot on the scintillator depended on the energy of the electrons. An image of energy variation as a function of time was created with a streak camera. The image from the streak camera was saved to a computer with a CCD camera. (See upper image in Figure 11.)

The data from the energy analyzer is compared with the computed gap voltage in Figure 11. The energy analyzer data include voltage variations caused by the injector, accelerator, and beam-induced voltage in the cells. Nonetheless, the gap-voltage and beam-energy have matching peaks and valleys for most of the beam. Beam locations with good matches are marked with a green dotted line. Mismatches are denoted by red lines. The first, left, red line does not have a match on the image because of the limited range of the analyzer. At about $40 \mathrm{~ns}$, the match is poor. This could be explained by a voltage drop in the injector, accelerator, and cell mis-timing after $40 \mathrm{~ns}$.

The beam current full-width half-maximum duration is about $72 \mathrm{~ns}$. The goal of the optimization effort is to produce an electronic beam with a "flat-top" that is $60 \mathrm{~ns}$ long with an energy variation of less than $1 \%$-rms. The energy analyzer indicated improvements were necessary.

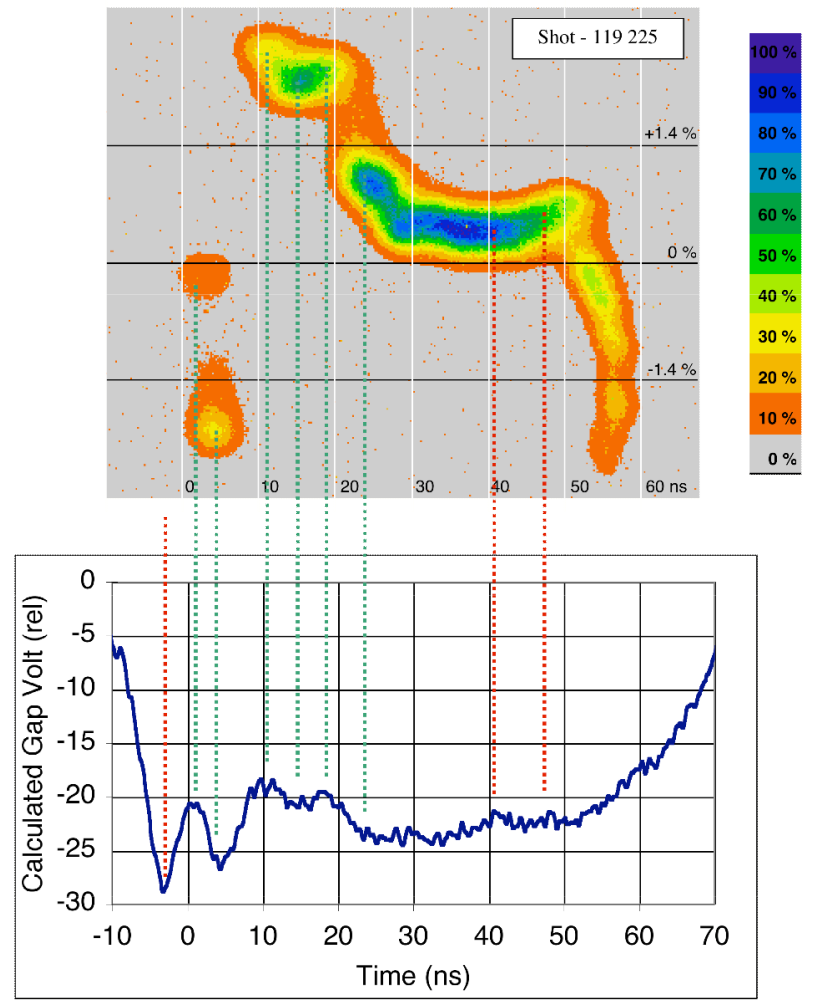

Figure 11. The image from an energy analyzer shows a similar pattern from the computed gap voltage for the most of the beam. 
(2) Beam loading can be estimated by comparing the pulse-power voltage in a cell with and without beam passing across the gap. FXR cells have voltage monitors located between the pulse-power feed and the oil / vacuum insulator. The top $10 \%$ of the voltage waveforms are shown in Figure 12.

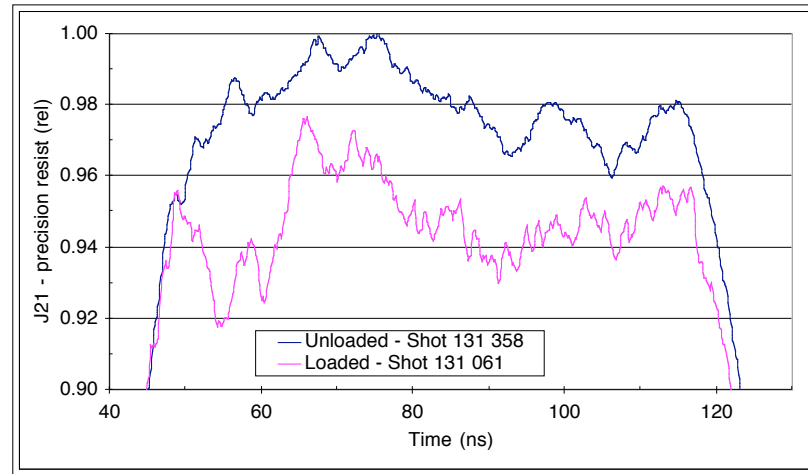

Figure 12. Cell voltage with and without beam loading.

The difference of the two waveforms is the beam loading voltage. This measured difference and the computed gap voltage is shown in Figure 13. The major features are very similar. They should not be the same because the cell voltage monitor is not located near the gap.
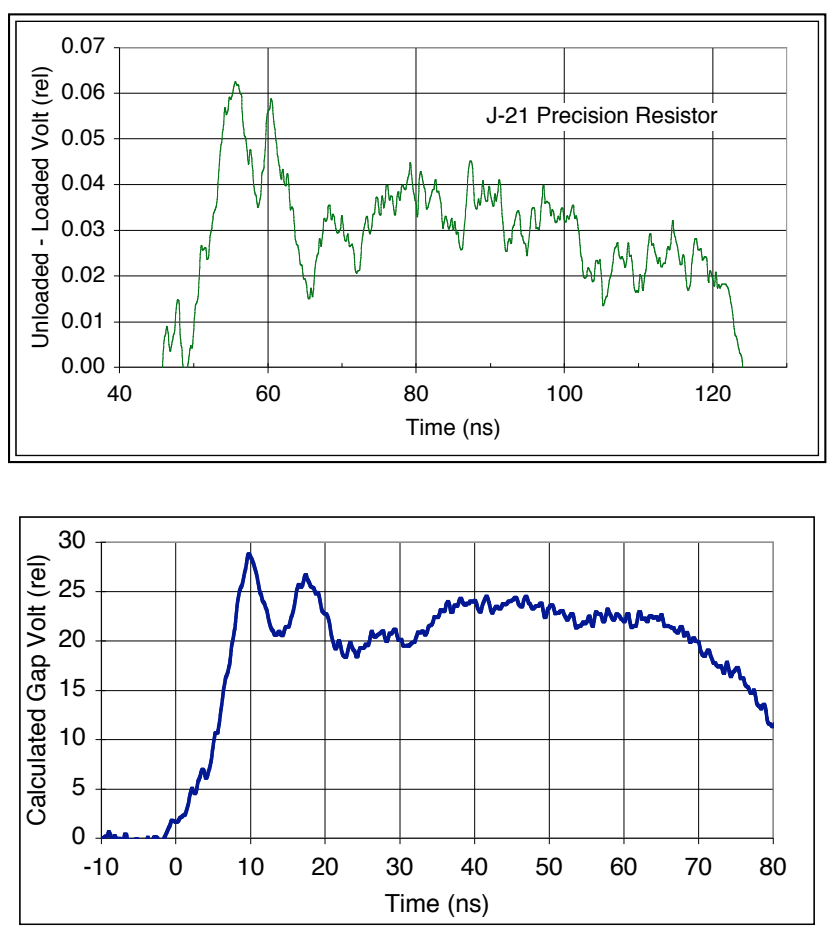

Figure 13. Measured beam loading voltage and computed beam-induced gap voltage have similar features.

(3) The previously mentioned cell voltage monitor was used to measure the beam-induced voltage by passing a beam across the gap with the pulse-power system off. The results from three cells located throughout the accelerator are shown in Figure 14 along with the computed gap voltage. Their trends are similar.

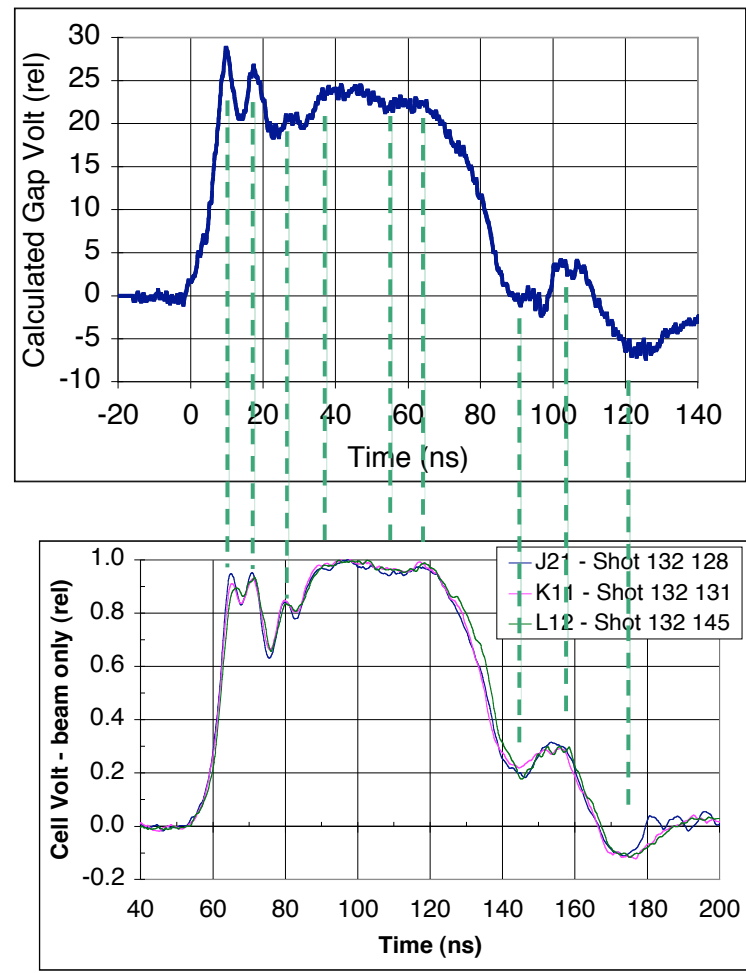

Figure 14. Computed gap voltage and measured beaminduced voltage have similar trends.

The agreement is extremely good in spite of the fact that the cell voltage monitor is not close to the gap. We believe that the computed gap voltage is a better predictor of beam energy variation caused by reflected electromagnetic waves in the cell.

The Test Stand has D-dot sensors installed near the gap to measure the electric field. When the dynamic beamsimulator shown in Figure 1 is completed, we will be able to further verify the computed gap voltage. It is difficult to install these D-dot sensors on FXR because the lack of space around our production accelerator.

The computed gap voltage based on the TDR measurements seem to be validated by the three types of measurements.

The effect on energy by the beam-induced cell voltage needs to be quantified. While it is possible to calculate the mean induced voltage and the standard deviation (or rms change around the mean) for a $60 \mathrm{~ns}$ beam, they may not be helpful measures. There is a complex interaction with injector energy and induced voltage. Because of this, a better measure is the mean cell impedance and rms variation.

Figure 15 shows the portion of the TDR measurement that is associated with the cell and pulse-power system. For the first $70 \mathrm{~ns}$, the mean impedance is $55.5 \Omega$, which is really $5.5 \Omega$ without the $50 \Omega$ air-line. The large variation is $2.3 \Omega$-rms and is driven mostly by the oscillations during the 
first $20 \mathrm{~ns}$. In the next section, we will examine methods for reducing the impedance change or voltage variation.

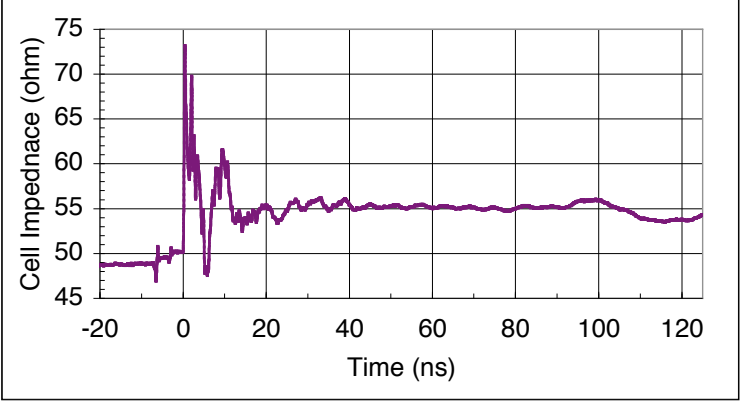

Figure 15. The cell and pulse-power system impedance has a mean value of $5.5 \Omega$ with a large variation of $2.3 \Omega$.

\section{Evaluation of Alternative Cell Designs}

Minimizing beam energy variations require compromises in accelerator and cell design. A large number of alternative designs are being studied including better control of the Marx voltage, flatter Blumlein pulse, better impedance matching of the components including the cell, longer ferrite operation, and reduced timing jitter. Three types of alternative design will be evaluated to determine their effect on the induced cell voltage: (1) slower rise-time beam (2) corner reflectors, and (3) different load resistance.

\section{Slow Rise-time Beam}

The oscillations in the cell impedance occur very quickly for the first $20 \mathrm{~ns}$. (See Figure 15.) By slowing down the rise-time of the beam, we can "average" out these faster impedance changes. The FXR beam rise-time is 9 ns. (See Figure 16.) If we slow the rise-time to $18 \mathrm{~ns}$, the induced voltage should be appreciably reduced.

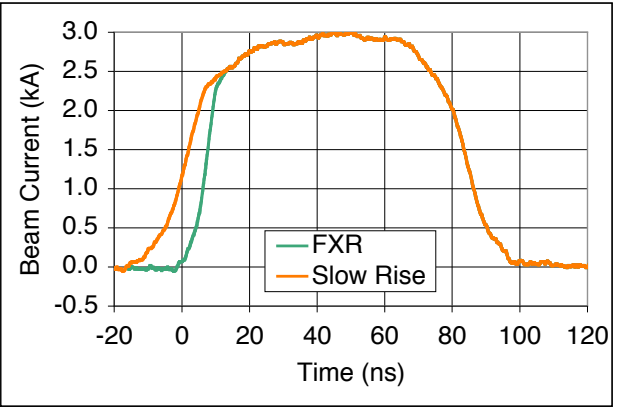

Figure 16. FXR beam rise-time is 9 ns shown in green, and the hypothetical slower beam has a rise-time of $18 \mathrm{~ns}$.

When the cell impedance is convolved with the slower rise-time current, the resulting variation in beam-induced voltage is much less for the first half of the beam. (See Figure 17.) The induced voltage has a longer duration. This is explained by the longer beam duration. (See Figure 18.)

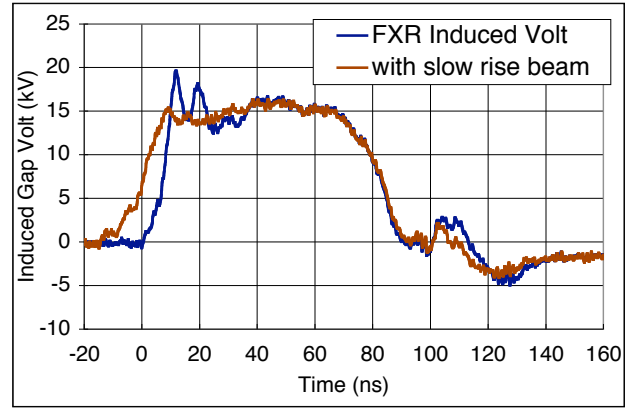

Figure 17. Computed beam-induced gap voltage for slower rise-time beam has less variation.

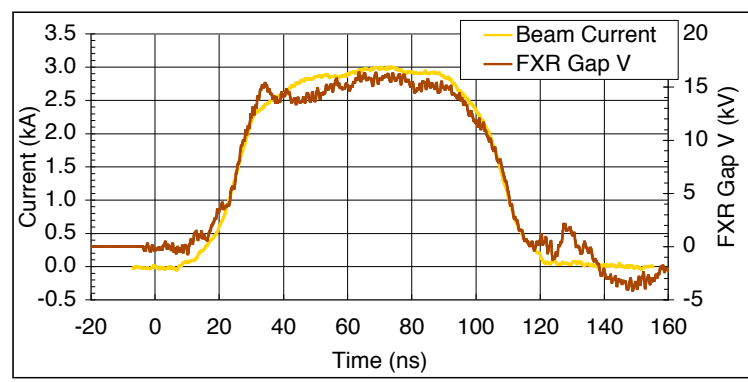

Figure 18. Waveforms of the slow rise-time beam current and induced gap voltage have the same duration.

Slowing down the rise-time of the beam may have detrimental effects on x-ray spot-size. The head of the beam has more off-energy electrons, and the result would be larger low-dose "wings" or ring around the main x-ray spot. The slower beam may also be more difficult to transport.

\section{Corner Reflectors}

Three different corner reflectors are being evaluated for their effect on beam-induced voltage: (1) current FXR design, (2) advanced reflector with $10 \Omega$ impedance, and (3) another advanced design with $6 \Omega$ impedance.

(1) The original corner reflector design is shown in pink in Figure 19. These corner reflectors were put into about half of the FXR cells to reduce BBU, beam breakup instability [4]. They shifted slightly the dominant BBU frequency, and hence slightly reduced the " $Q$ " of the composite accelerator cells.
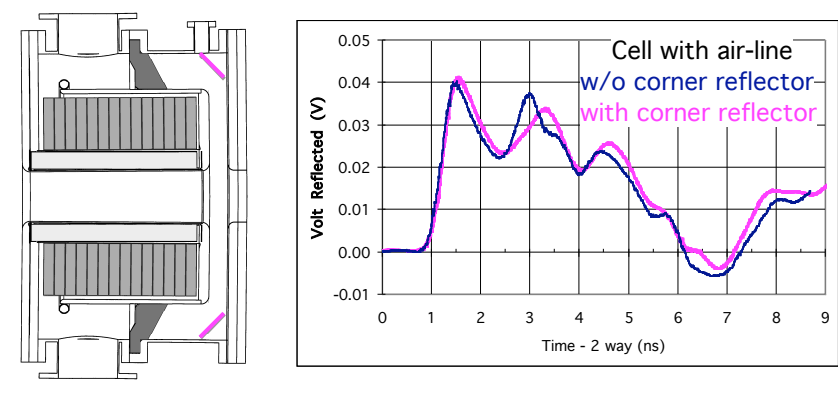

Figure 19. TDR measurement of FXR cell with (denoted in pink) and without (blue) corner reflector. 
The reflected voltage from the TDR measurement shows a slightly lower value for the second peak associated with the corner. It is also slightly later in time. This slight change in voltage or impedance will not have a significant effect on the beam-induced voltage. Therefore, an advanced corner reflector was designed to reduce the corner reflections.

(2) The objective of the first advanced corner reflector was to reduce the impedance at the corner to the insulator to a constant $10 \Omega$ from the current maximum value of around $20 \Omega$. The $10 \Omega$ level would match the pulse-power system transmission-line impedance. Another objective was to reduce $\mathrm{BBU}$ by directing more energy back into the ferrites, load resistors and pulse-power system.

The first advanced corner reflector is shown in Figure 20. The holes in the ring allow the vacuum pumps to remove any trapped air between the reflector and the corner of the cell.

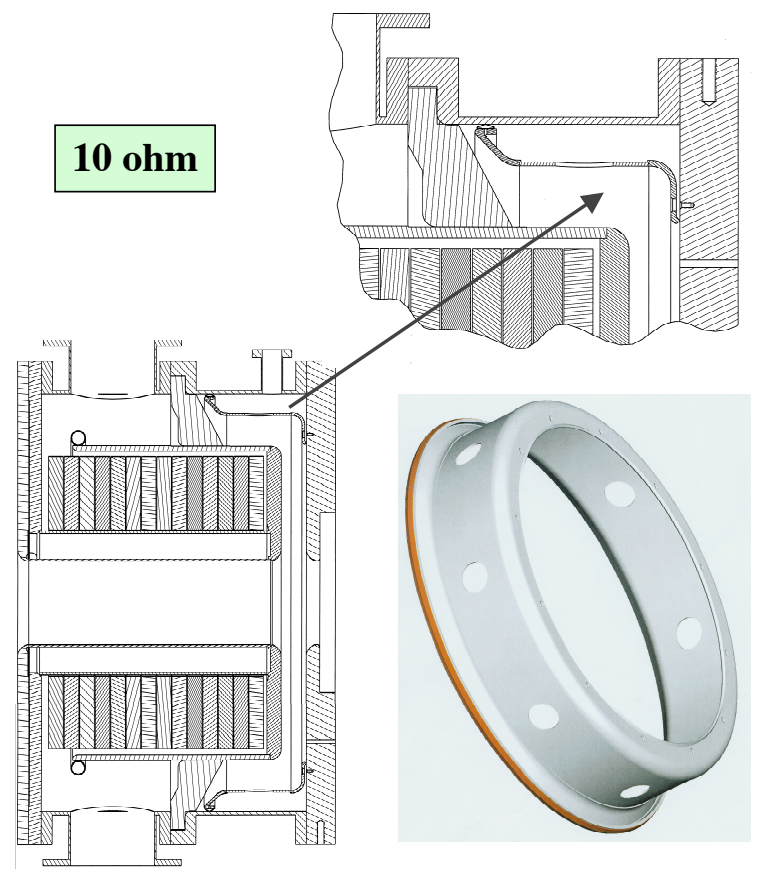

Figure 20. Advance corner reflector with $10 \Omega$ impedance.

For the computer simulation, the impedance of the cell from the corner to the insulator was reduced to $10 \Omega$. (See Figure 21.)

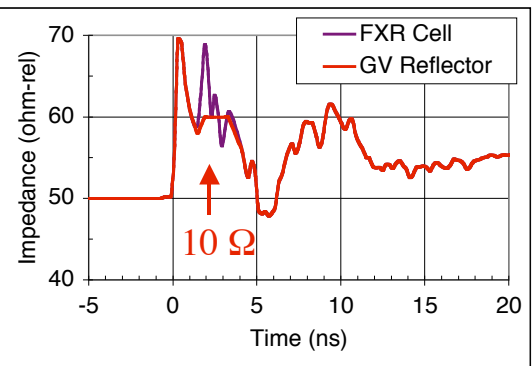

Figure 21. For the computer simulation, the impedance was reduced at the advanced corner reflector location.
The reduction in variation of the beam-induced voltage was small. (See Figure 22.) The rise-time of the beam is 9 ns, and the average cell impedance over this interval was not significantly reduced with the proposed corner reflector. The waveforms of the beam current and computed gap voltage are overlaid in Figure 23. A more aggressive design was considered.

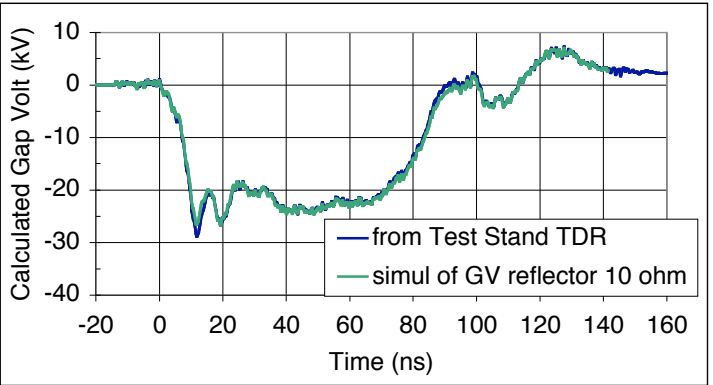

Figure 22. Computed beam-induced gap voltage for the standard FXR cell and with first advanced corner reflector showed small improvement.

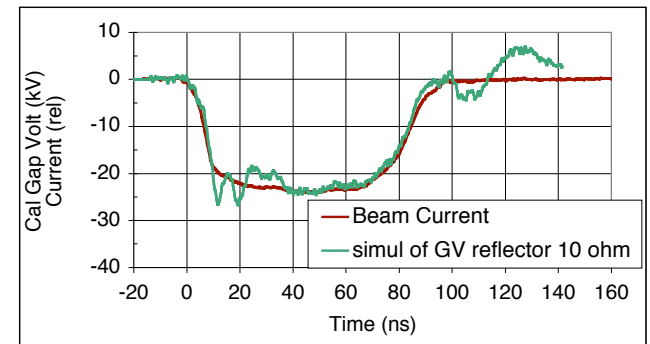

Figure 23. Beam current and computed gap voltages have the same duration.

(3) In theory, "steady-state" impedance of the cell and pulse-power system is 7.2 $\Omega$. The TDR measurements produced a slightly lower impedance of $6 \Omega$. This value is within the accuracy of the instrument because the total measured impedance was $56 \Omega$. For the computer simulation, the impedance in the area of the second advanced corner reflector was reduced to $6 \Omega$. (See Figure 24.)

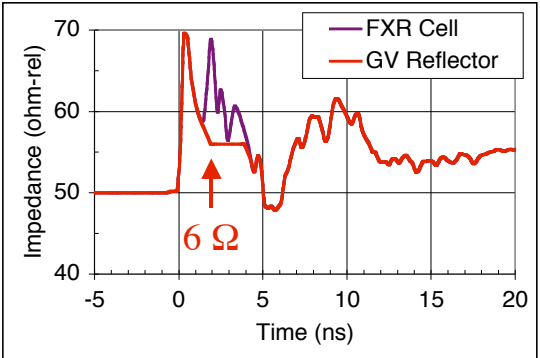

Figure 24. For the computer simulation, the corner impedance was reduced further to $6 \Omega$.

The computer simulation gap voltage shows a significant reduction in the variation at the first peak. (See Figure 25.) The beam current and gap voltage is overlaid in Figure 26. As expected, the gap voltage follows more closely the 
current profile at the first peak when compared with the waveforms in Figure 23. It may not be possible to reduce the impedance in the space between the corner and insulator by inserting a reflector. The electric fields will be increased at the corner, and electron emission might occur.

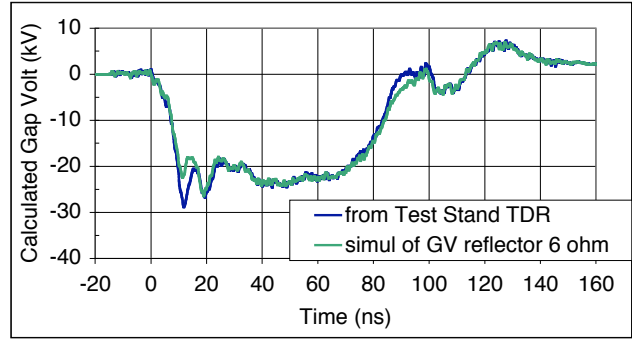

Figure 25. A $6 \Omega$ corner reflector would reduce the first peak of the beam-induced voltage.

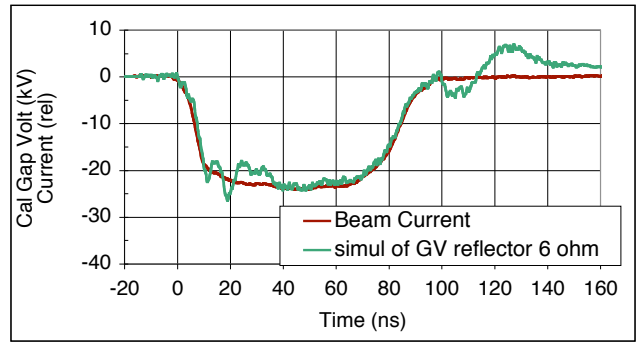

Figure 26. The gap voltage follows more closely the current profile at the first peak.

The advanced corner reflectors are being designed and fabricated. TDR measurements will be made, and highvoltage studies will be performed on the Test Stand.

(3) The pulse-power system has a combined output impedance of $10.7 \Omega$. The two cell load resistors are $44 \Omega$ each, or $22 \Omega$ in parallel, and therefore there is an impedance mismatch. This was needed to increase the voltage at the cells.

Our cell voltage has a bump towards the end of the acceleration pulse. From field measurements taken in the water-line of the Test Stand, we believe that a positive reflection is created at the higher impedance cell. It propagates back to the Blumlein where it is again reflected. Because the water-line is not long enough, this second reflection adds to the end of the original pulse.

To reduce the reflections, the load resistors were changed to $22 \Omega$ so that the pulse-power system saw a matched load. The cell and pulse-power system impedance measured from the gap with the TDR instrument is shown in Figure 27.

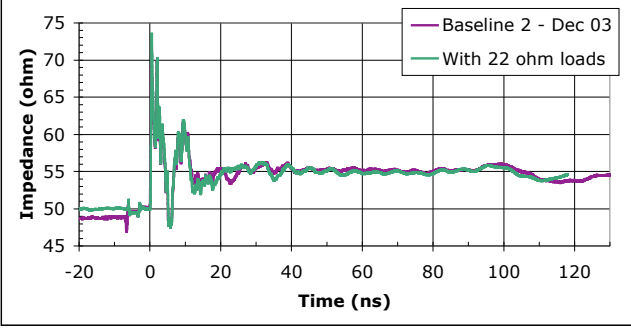

Figure 27. Reducing the load resistor reduces the "steadystate" cell and pulse-power system impedance.

The "steady-state" impedance should have been reduced from $7.2 \Omega$ to $5.4 \Omega$. The TDR results show less reduction, but are probably within the error-bar of the instrument.

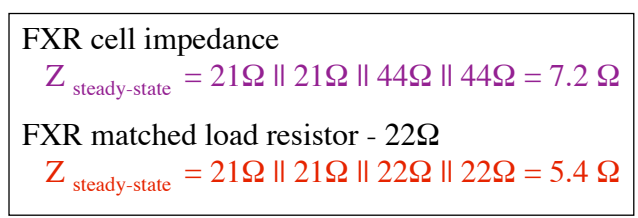

While the impedance matching may make the pulsepower voltage more stable, it creates a couple of problems. This lower impedance could make injector energy variation compensation somewhat less effective. This subject is covered in another report. The variation in beam-induced voltage will also increase slightly because the peaks will be relatively higher than the "steady-state" voltage. (See Figure 28.) Accelerator optimization requires evaluating many trade-offs.

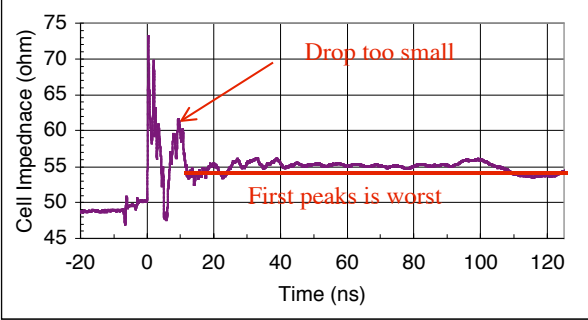

Figure 28. Decreasing the "steady-state" impedance will increase the voltage variation in the beam-induced voltage.

We have also tried improving the impedance match between the oil and water interface near the cell feed. This plastic interface is only a couple of inches long, and the change would only minimally alter the beam-induced voltage.

The real problem with the beam-induced voltage is the electromagnetic waves reflecting in and around the cell. A traditional approach to solving this problem is with RF absorbing materials. They will be evaluated with guidance from 3D EM modeling of the cell.

The variation in beam-induced voltage is only a piece of the x-ray spot-size puzzle. Beam emittance and voltage variations from other accelerator components must be considered in making technical, cost and schedule trade-offs. The results from this study on induced voltages will be 
incorporated into a larger accelerator system-model to quantify their effect on total beam energy variations.

\section{Acknowledgments}

We want to acknowledge the contributions of Blake Kreitzer, Jim Bowman, Ken Griffin and other mechanical and electronic personnel who worked hard to build the Test Stand and support the TDR measurements. The ideas for improving voltage regulations came from many people including Ron Kihara, Dave Goerz, and Bill DeHope. We want to especially thank B-Program, Dave Goerz and Ray Scarpetti for funding this work.

\section{References}

[1] Multhauf, L.G., "The LLNL Flash X-ray Induction Linear Accelerator", LLNL, Livermore, CA, UCRL-JC148543, Sept 19, 2002.

[2] Ong, Mike, George Vogtlin, Dave Goerz and Ray Scarpetti, "Flash X-Ray (FXR) Accelerator Optimization", $14^{\text {th }}$ IEEE International Pulsed Power Conference, Dallas, TX, June 2003, pp. 909-12.

[3] Ong, Mike and Jan Zentler, "Electron Energy Spectrum Measurement Single Pulse”, May 2000, FXR Note 045, Category 7, Lawrence Livermore National Laboratory.

[4] Ong, Mike, Carlos Avalle, Roger Richardson, Jan Zentler, "LLNL Flash X-ray Radiography Machine (FRX) Double-pulse Upgrade Diagnostics", $11^{\text {th }}$ IEEE International Pulsed Power Conference, Baltimore, MD, June 1997, pp. 430-5. 